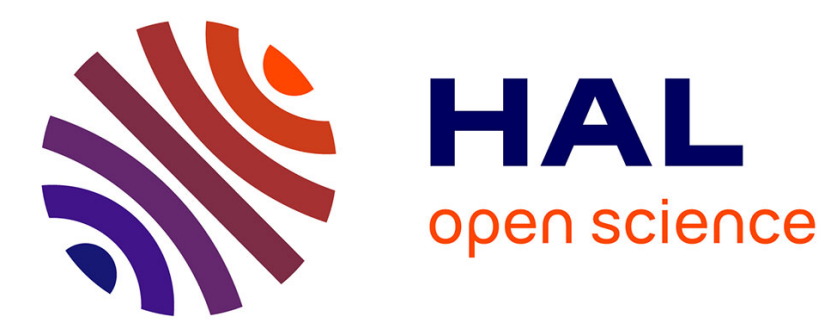

\title{
Stability and Index of the Meet Game on a Lattice
}

Joseph M. Abdou

\section{To cite this version:}

Joseph M. Abdou. Stability and Index of the Meet Game on a Lattice. International Journal of Game Theory, 2012, 41 (4), pp.775-789. 10.1007/s00182-012-0339-5 . halshs-00633589

\section{HAL Id: halshs-00633589 \\ https://shs.hal.science/halshs-00633589}

Submitted on 18 Oct 2011

HAL is a multi-disciplinary open access archive for the deposit and dissemination of scientific research documents, whether they are published or not. The documents may come from teaching and research institutions in France or abroad, or from public or private research centers.
L'archive ouverte pluridisciplinaire HAL, est destinée au dépôt et à la diffusion de documents scientifiques de niveau recherche, publiés ou non, émanant des établissements d'enseignement et de recherche français ou étrangers, des laboratoires publics ou privés. 


\title{
Stability and Index of the Meet Game on a Lattice
}

\author{
Joseph M. Abdou *†
}

June 17, 2011

\begin{abstract}
We study the stability and the stability index of the meet game form defined on a meet semilattice. Given any active coalition structure, we show that the stability index relative to the equilibrium, to the beta core and to the exact core is a function of the Nakamura number, the depth of the semilattice and its gap function.
\end{abstract}

Keywords: Effectivity Function, Lattice, Stability Index, Equilibrium, Nakamura Number.

JEL Classification: C70, D71 AMS Classification: 91A44

${ }^{*}$ Centre d'Economie de la Sorbonne (CES) CNRS : UMR8174 - Université Panthéon-Sorbonne Paris I.

${ }^{\dagger}$ École d'Économie de Paris - Paris School of Economics (EEP-PSE), email: abdou@univ-paris1.fr 


\section{Introduction}

Stability is an essential requirement for political institutions; however it is both a theoretical and an empirical fact that political institutions are often unstable. Instability occurs when contradictory forces prevent the emergence of a persistent outcome, that is a state that is self-sustained and defendable when it is subject to attacks aiming to dismantle it. What can be observed when instability occurs is a volatile situation: any outcome that is proposed is subject to obstruction by some factions that block it. The result is a stalemate, where the institution is deadlocked. As far as the governance or the constitution of an established state is concerned, such a deadlock paves the way for a dramatic change of the institution itself; the latter is necessarily exogenous since no endogenous solution can be expected. In [2] and [3] the author argues that, however chaotic it may seem at first sight, instability in regulated systems presents some regularity and therefore there exist "patterns of instability".

Our basic model for analyzing interactions consists of a strategic game form $G$ and some solution concept say $\mathcal{E}$. We shall say that such a mechanism is stable if it has solutions for all preference profiles. The absence of solution results from conflicting relations between preferences of the agents and the opposition power that the game form allocates to active agents or coalitions. If groups of alternatives are merged together, the power distribution being preserved, then preferences become comparatively smoothed and a solution may emerge. Merging alternatives amounts to making a partition of the alternative set in such a way that agents are indifferent between elements of the same class. The minimal size of such a partition with no solution is the stability index. It is well known $([2][3])$ that instability for the pair $(G, \mathcal{E})$ is closely related to the existence of some generalized Condorcet cycle, and that the stability index is equal to the minimal size of such a cycle. In [2] some general properties related to the structure of instability have been shown for the core and the exact core. In [3] the study has been extended to Nash-like solutions including Nash proper and strong Nash.

In this paper we characterize stability and we compute the stability index of the meet game form. Let $(A, \wedge)$ be a meet semilattice. The $n$-player meet game form on $A$ is defined as follows: each player chooses $x_{i} \in A$, the outcome is given by $\mu\left(x_{1}, \ldots, x_{n}\right)$ $=x_{1} \wedge \cdots \wedge x_{n}$. While this game form can be hardly favored by a social engineer, or defended on philosophical or normative grounds ${ }^{1}$, it may represent a simple model for static interactive pollution. If every player selects a vector of pollution levels $\left(\mathrm{CO}_{2}\right.$, radiation, etc ...) represented by some vector in $\mathbb{R}^{d}$, then the resulting outcome is the infimum (or the supremum) of individual vectors.

It appears from [2] and [3], that given some game form, an effective calculation of the stability index for the core is not an easy matter, let alone the stability index for Nash equilibrium. Here, the particular game form that we propose allows us to carry out such calculations. Given the importance of the subject for political systems, we hope that the neatness of the results presented in this paper beyond its technical difficulties

\footnotetext{
${ }^{1}$ As a referee of an earlier version of this paper put it: this is not one which would come up immediately in connection with any realistic problem of social choice. Initially, my choice was motivated mainly by practical reasons, which is why I did not try to justify it on social choice grounds. However, on a second thought, this game form, with only little exaggeration is the de facto decision rule for a wide range of European Union issues.
} 
and the modesty of its scope provides an invitation to future more comprehensive research on this problem.

Let $\mathcal{M}$ be any subset of non empty coalitions. Solutions that are considered in this paper are either the $\beta$-core, or the exact-core or Nash-like equilibrium where only coalitions in $\mathcal{M}$ are active. It turns out, that stability and the stability index depend on three parameters: On the side of the players the Nakamura number or $\nu_{\mathcal{M}}$, and on the side of the alternative set, the depth of $A$ or $\delta_{A}$ and the gap function or $\gamma_{A}$.

In the first section we present the stability problem as well as the theoretical tools for the analysis of the stability index. Section 2 is devoted to the meet game form on a semilattice. In particular two notions are defined for any semilattice: the depth and the gap functions. They play an essential role along with the Nakamura number in the characterization of stability and the stability index. Moreover section 2 shows that all what is needed for our purpose is to know the local effectivity function of the meet game form. In section 3 we prove the main results on stability and stability index of the meet game form. Some technical proofs relative to binary relations are the object of an appendix.

\section{Game forms}

\section{$1.1 \quad$ Notations}

Throughout this paper we shall consider a finite set $N=\{1, \ldots, n\}$ the elements of which are called players, and a finite set $A=\left\{a_{1}, \ldots, a_{p}\right\}$ the elements of which are called alternatives. We make use of the following notational conventions: For any set $X$, we denote by $\mathcal{P}(X)$ the set of all subsets of $X$ and by $\mathcal{P}_{0}(X)=\mathcal{P}(X) \backslash\{\emptyset\}$ the set of all non-empty subsets of $X . Q(X)$ (resp. $L(X)$ ) will denote the set of all preorders (resp. linear orders) on $X$, that is all binary relations on $X$ which are transitive and complete (resp. transitive, complete and antisymmetric). If $R \in Q(X)$ we denote by $R^{\circ}$ (resp. $R^{\sim}$ ) the strict binary relation (resp. the equivalence relation) induced by $R$ on $X$. Elements of $\mathcal{P}_{0}(N)$ are called coalitions. If $S \in \mathcal{P}_{0}(N)$ then $N \backslash S$ is denoted $S^{c}$. Similarly if $B \in \mathcal{P}(A), A \backslash B$ is denoted $B^{c}$. A preference profile (over $A$ ) is a map from $N$ to $Q(A)$, so that a preference profile is an element of $Q(A)^{N}$. For every preference profile $R_{N} \in Q(A)^{N}$ and $S \in \mathcal{P}_{0}(N)$ we put

$$
P\left(a, S, R_{N}\right)=\left\{b \in A \mid b R_{i}^{\circ} a, \forall i \in S\right\}
$$

(so that $P\left(a, S, R_{N}\right)$ consists of all the outcomes considered to be strictly better than $a$ by all members of coalition $S)$.

\subsection{Solutions, stability and index}

Let $G=\left\langle X_{1}, \ldots, X_{n}, A, g\right\rangle$ be a strategic game form. The set of players is $N=$ $\{1, \ldots, n\}, X_{i}$ is the strategy set of players $i, g: \prod_{i \in N} X_{i} \rightarrow A$ is the outcome function, assumed to be surjective. For any $S \in \mathcal{P}_{0}(N)$ the product $\prod_{i \in S} X_{i}$ will be denoted $X_{S}$. Given any preference profile $R_{N} \in Q(A)^{N}$, the game form $G$ induces a game $\left(X_{1}, \ldots, X_{n} ; Q_{1}, \ldots, Q_{n}\right)$ with the same strategy spaces and where $Q_{i}$ is the preorder on $X_{N}$ defined by: $x_{N} Q_{i} y_{N}$ if and only if $g\left(x_{N}\right) R_{i} g\left(y_{N}\right)$ for $x_{N}, y_{N} \in X_{N}$. We denote this game by $G\left(R_{N}\right)$. 
For our solution concepts we shall assume that only some coalitions can form. Any $\mathcal{M} \subset \mathcal{P}_{0}(N)$ is called an active coalition structure. The first solution concept is similar to Nash equilibrium. It has been introduced in [8] (definition 5.1.6):

A strategy array $x_{N} \in X_{N}$ is an $\mathcal{M}$-equilibrium of the game $G\left(R_{N}\right)$ if there is no coalition $S \in \mathcal{M}$ and $y_{S} \in X_{S}$ such that $g\left(y_{S}, x_{S^{c}}\right) R_{i}^{\circ} g\left(x_{N}\right)$ for all $i \in S$. An alternative $a$ is an $\mathcal{M}$-equilibrium outcome of $G$ at $R_{N}$ if there exists some equilibrium $x_{N} \in X_{N}$ of $G\left(R_{N}\right)$ such that $g\left(x_{N}\right)=a$. We denote by $E O(\mathcal{M})\left(G, R_{N}\right)$ the set of all $\mathcal{M}$-equilibrium outcomes of $\left(G, R_{N}\right)$. In particular, when $\mathcal{M}=\mathcal{N} \equiv\{\{1\}, \ldots,\{n\}\}$, an $\mathcal{M}$-equilibrium is a Nash equilibrium. Similarly, when $\mathcal{M}=\mathcal{P}_{0}(N)$, an $\mathcal{M}$-equilibrium is a strong Nash equilibrium.

The following solutions have been defined respectively in [1] and [6]:

An alternative $a$ is in the $\mathcal{M}$-exact core of $\left(G, R_{N}\right)$ if there is no coalition $S \in \mathcal{M}$ with the following property : for any $z_{N} \in X_{N}$ such that $g\left(z_{N}\right)=a$ there exists $y_{S} \in X_{S}$ such that $g\left(y_{S}, z_{S^{c}}\right) R_{i}^{\circ} g\left(z_{N}\right)$ for all $i \in S$. Denote by $C_{1, \mathcal{M}}\left(G, R_{N}\right)$ the $\mathcal{M}$-exact core of $\left(G, R_{N}\right)$.

An alternative $a$ is in the $\mathcal{M}$ - $\beta$-core of $\left(G, R_{N}\right)$ if there is no coalition $S \in \mathcal{M}$ with the following property: for any $z_{N} \in X_{N}$, there exists $y_{S} \in X_{S}$ such that $g\left(y_{S}, z_{S^{c}}\right) R_{i}^{\circ} a$ for all $i \in S$. Denote by $C_{0, \mathcal{M}}\left(G, R_{N}\right)$, the $\mathcal{M}$ - $\beta$-core of $\left(G, R_{N}\right)$.

Let $\Pi$ be the set of all partitions of $A$. If $\pi \in \Pi$ and $a \in A$ we denote by $\pi(a)$ the class of the partition that contains $a$. Let $Q \bullet(\pi)$ be the set of all $R \in Q(A)$ such that whenever $\pi(a)=\pi(b)$ then $a R^{\sim} b$. Let $\pi, \pi^{\prime} \in \Pi$. $\pi$ refines $\pi^{\prime}$, if every element of $\pi$ is included in some element of $\pi^{\prime}$. If $\pi$ refines $\pi^{\prime}$ then $Q_{\bullet}(\pi) \subset Q_{\bullet}\left(\pi^{\prime}\right)$.

Let $\Pi_{r}$ be the set of all partitions of $A$ with $r$ elements. Since any element of $\Pi_{r}$ has a refinement in $\Pi_{r+1}$, then $\left\{Q_{\bullet}(\pi): \pi \in \Pi_{r}\right\} \subset\left\{Q_{\bullet}(\pi): \pi \in \Pi_{r+1}\right\}$ for any $r \geq 1$. Moreover $\left\{Q_{\bullet}(\pi) \mid \pi \in \Pi_{1}\right\}$ is a singleton and $\left\{Q_{\bullet}(\pi) \mid \pi \in \Pi_{p}\right\}=Q(A)$. We say that $G$ is $r$-M-solvable if $\operatorname{EO}(\mathcal{M})\left(G, R_{N}\right) \neq \emptyset$ for all $R_{N} \in Q_{\bullet}(\pi)^{N}$ and all $\pi \in \Pi_{r} . G$ is $r$-M-exactly stable if $C_{1, \mathcal{M}}\left(G, R_{N}\right) \neq \emptyset$ for all $R_{N} \in Q \bullet(\pi)^{N}$ and all $\pi \in \Pi_{r} . G$ is $r$-M- $\beta$-stable if $C_{0, \mathcal{M}}\left(G, R_{N}\right) \neq \emptyset$ for all $R_{N} \in Q \bullet(\pi)^{N}$ and all $\pi \in \Pi_{r}$. We say that $G$ is $\mathcal{M}$-solvable if $G$ is $r$ - $\mathcal{M}$-solvable for all $r \geq 1$. Similar definitions can be made for the $\mathcal{M}$-exact core and the $\mathcal{M}$ - $\beta$-core.

Definition 1.1 The stability index of $G$ relatively to the $\mathcal{M}$-equilibrium (resp. $\mathcal{M}$ exact core, resp. $\mathcal{M}$ - $\beta$-core) is the smallest integer $r \geq 1$ such $G$ is not $r$ - $\mathcal{M}$-solvable (resp. $r$ - $\mathcal{M}$-exactly stable, $r$ - $\mathcal{M}$ - $\beta$-stable) (with the convention that the index is $+\infty$ if no such integer exists).

When it is finite, the stability index is an integer that can take values ranging from 2 to $p$ ( $p$ is the number of elements of the alternative set) and it depends on the pair composed of the game form and the solution concept. This index defines a threshold on the sophistication level of a society that can safely use the game form (ruled by that solution). If stability is out of reach, the social engineer is led to recommend institutions with a rather high stability index. Indeed we shall see below that this number is equal to the length of some generalized Condorcet cycle and it has been argued in [2] and [3] that longer cycles are more costly than shorter ones and therefore a game form with a higher index is less likely to incur instability. In many countries, the society splits over two main issues. There are two main parties. Each party can 
defend one issue but cannot force that issue. The result is a typical deadlock that, in our theory would be represented by an interaction with stability index equal to 2 . Empirically, countries with ethnic or religious division are often deadlocked by such a picture. Violence is the likely conclusion of the stalemate. On the other hand a typical situation with index 3 allows some governance (if we take time into consideration), but such a governance is precarious since any alliance of two parties can be threatened by a third party that eventually succeeds in breaking the alliance, only to fall in a new precarious state. More generally the study of cycle formation sheds a light on the form of instability that threatens a political interaction.

The theoretical aspects of the stability index have been exposed in [2] and [3]. In the first paper only the stability index of the exact core and the $\beta$-core has been defined and computed. It turns out that, for the mentioned solutions, the stability index is 2,3 or $+\infty$ (for more details see [2] Proposition 4.18). When it comes to Nash equilibrium and strong Nash equilibrium, some general result can be found in [3], but the study points out the difficulty to calculate the Nash stability index except for some special classes of game forms (e.g. rectangular game forms). A second difficulty arises from the fact that in the case of Nash-like equilibrium solutions, effectivity functions are not appropriate to the description of the the underlying power, and one is led to define more complex objects for that purpose. By contrast, the game form that we study in this paper has the property that only the local effectivity functions is required (see theorems 2.7 and 2.8 below) and thus the calculations can be carried out to the conclusion.

The object of this paper is to give necessary and sufficient conditions for stability and to determine the stability index of the following game form, called the meet game form $\Gamma=\left\langle X_{1}, \ldots, X_{n}, A, \mu\right\rangle$, where $X_{1}=\cdots=X_{n}=A, A$ is a meet-semilattice (precise definitions are given below), and $\mu$ is the meet function that is:

$$
\mu\left(x_{1}, \ldots, x_{n}\right)=x_{1} \wedge \cdots \wedge x_{n} \quad\left(x_{1} \in A, \ldots, x_{n} \in A\right) .
$$

The method that we follow to characterize stability and to calculate the stability index is based on the distribution of power that underlies the solution concept. As we shall see, for such a power distribution, instability takes the familiar form of the existence of some Condorcet-like cycle. The stability index is closely related to the minimal length of a cycle. The power distribution that we need is known as the local effectivity function.

\subsection{Power distribution, effectivity structure and cycles}

Definition 1.2 A local effectivity function on $(N, A)$ is a family $E \equiv(E[U], U \in$ $\left.\mathcal{P}_{0}(A)\right)$ where for any $U \in \mathcal{P}_{0}(A), E[U]: \mathcal{P}(N) \rightarrow \mathcal{P}\left(\mathcal{P}_{0}(A)\right)$ and such that the following conditions are satisfied:

(i) $E[U](\emptyset)=\emptyset$,

(ii) $B \in E[U](S), B \subset B^{\prime} \Rightarrow B^{\prime} \in E[U](S)$,

(iii) $U \subset V \Rightarrow E[V](S) \subset E[U](S)$.

A local effectivity function is an effectivity function if it does not depend on $U$.

The formula $B \in E[U](S)$ is interpreted as follows: When the current state is in $U$, 
coalition $S$ can threaten to achieve some state in $B$. Let $R_{N} \in Q(A)^{N}$. An alternative $a \in A$ is dominated at $R_{N}$ if there exists $U \in \mathcal{P}_{0}(A), S \in \mathcal{P}_{0}(N)$ such that $a \in U$ and $P\left(a, S, R_{N}\right) \in E[U](S)$. The core of $E$ at $R_{N}$ is the set of undominated alternatives. It is denoted $C\left(E, R_{N}\right)$. We say that $E$ is $r$-stable if $C\left(E, R_{N}\right) \neq \emptyset$ for all $R_{N} \in Q \bullet(\pi)^{N}$ and all $\pi \in \Pi_{r}$. We say that $E$ is stable if $E$ is $r$-stable for all $r \geq 1$. The stability index of $E$ is the minimal integer $r$ such that $E$ is not $r$-stable (with the convention that this index is $+\infty$ if $E$ is stable). It will be denoted $\sigma(E)$.

Let $G$ be a strategic game form. We are now ready to determine the underling power distribution in $G$ relatively to the exact core and the $\beta$-core. For any coalition $S$ and any alternative $a$, the idea is to describe the retaliation power of $S$ if the current issue is $a$. This will be represented by the set of all $B \subset A$ that satisfy the following property: if $g(x)=a$, then for some strategy $y_{S} \in X_{S}, g\left(x_{S^{c}}, y_{S}\right) \in B$. This set is therefore the following:

$$
E_{1, \mathcal{M}}^{G}[a](S)=\left\{B \in \mathcal{P}_{0}(A) \mid \forall x_{N} \in g^{-1}(a), \exists y_{S} \in X_{S}: g\left(x_{S^{c}}, y_{S}\right) \in B\right\}
$$

The local effectivity function $E_{1, \mathcal{M}}^{G}$ associated to $(G, \mathcal{M})$ is defined as follows: For $U \in \mathcal{P}_{0}(A), S \notin \mathcal{M}: E_{1, \mathcal{M}}^{G}[U](S)=\emptyset$, and for $S \in \mathcal{M}$ :

$$
E_{1, \mathcal{M}}^{G}[U](S)=\cap_{a \in U} E_{1, \mathcal{M}}^{G}[a](S)
$$

The $\beta$-effectivity function associated to $(G, \mathcal{M})$ is defined by $E_{0, \mathcal{M}}^{G}(S)=E_{1, \mathcal{M}}^{G}[A](S)$ $(S \in \mathcal{P}(N))$.

Lemma 1.3 The $\mathcal{M}$-exact core (resp. $\mathcal{M}$ - $\beta$-core) of $\left(G, R_{N}\right)$ coincides with the core of $E_{1, \mathcal{M}}^{G}$ (resp. $E_{0, \mathcal{M}}^{G}$ ) at $R_{N}$. Therefore $G$ is $r$-M-exactly stable (resp. $r$ - $\mathcal{M}$ - $\beta$-stable) if and only if $E_{1, \mathcal{M}}^{G}$ (resp. $E_{0, \mathcal{M}}^{G}$ ) is r-stable. In particular the stability index relatively to the $\mathcal{M}$-exact core of $G$ is equal to the stability index of $E_{1, \mathcal{M}}^{G}$.

Proof. Straightforward.

In general the power distribution relative to $\mathcal{M}$-equilibrium exceeds the local effectivity function and requires more complex effectivity structures ([1]). However, we shall see, that in the case of the meet game form, the local effectivity function does the job (Proposition 2.1). The existence of a preference profile with an empty exact core is equivalent to the existence of some combinatorial configuration that can be viewed as a generalized Condorcet cycle. Such a configuration has been introduced in [5] for effectivity functions, and in [4] to more general effectivity structures. The following definition, that can be found in [2] is an adaptation of the definition to local effectivity functions.

Definition 1.4 Let $E$ ba a local effectivity function. An $r$-tuple $\left(\left(C_{1}, B_{1}, S_{1}\right), \ldots\right.$, $\left.\left(C_{r}, B_{r}, S_{r}\right)\right)$ where $r \geq 1, C_{k} \in \mathcal{P}_{0}(A), B_{k} \in \mathcal{P}_{0}(A), S_{k} \in \mathcal{P}_{0}(N)(k=1, \ldots, r)$ is a dominance configuration of $E$ if:

(i) $B_{k} \in E\left[C_{k}\right]\left(S_{k}\right)(k=1, \ldots, r)$.

(ii) $\left(C_{1}, \ldots, C_{r}\right)$ is a partition of $A$. 
$\left(C_{1}, \ldots, C_{r}\right)$ is said to be the basis of the dominance configuration and $r$ its length or order.

A dominance configuration $\left(\left(C_{1}, B_{1}, S_{1}\right), \ldots,\left(C_{r}, B_{r}, S_{r}\right)\right)$ is a cycle of $E$ if it satisfies the following property :

(C) For any $\emptyset \neq J \subset\{1, \ldots, r\}$ such that $\cap_{k \in J} S_{k} \neq \emptyset$, there exists $\ell \in J$ such that for all $k \in J: C_{\ell} \cap B_{k}=\emptyset$.

There are other equivalent ways to express property $(\mathrm{C})$ :

(C') For any $\emptyset \neq J \subset\{1, \ldots, r\}$ such that $\cap_{k \in J} S_{k} \neq \emptyset$, there exists $\ell \in J$ such that for all $k \in J: B_{\ell} \cap C_{k}=\emptyset$.

(C") For any sequence $k_{1}, \ldots, k_{s}$ of elements in $\{1, \ldots, r\}$ either $\cap_{t=1}^{s} S_{k_{t}}=\emptyset$ or $\left[U_{k_{1}} \cap\right.$ $\left.B_{k_{2}} \neq \emptyset, \ldots, U_{k_{t}} \cap B_{k_{t+1}} \neq \emptyset, \ldots, U_{k_{s}} \cap B_{k_{1}} \neq \emptyset\right]$

Properties $(C)$ and $\left(C^{\prime}\right)$ are dual. Property $\left(C^{\prime \prime}\right)$ was originally introduced by Keiding [5] in the context of effectivity functions. The equivalence of these properties is easy to prove. Initially Keiding [5] proved that stability is equivalent to the absence of cycles. This result was extended to local effectivity functions in Abdou [2], and indeed to a larger framework in Abdou and Keiding [4]. Here we recall the result for local effectivity functions:

Theorem 1.5 A local effectivity function is stable if and only if it has no cycles.

While existence of a cycle implies instability, the determination of the stability index requires a deeper knowledge of the structure of the effectivity structure. One is supposed to find a partition $\pi$ of smallest cardinality such that the core is empty for some $R_{N} \in Q \cdot(\pi)^{N}$. For that purpose, the following characterization shows that it is equivalent to determine the minimal length of a cycle. Indeed, in view of Abdou [2] Theorem 4.4, we have:

Theorem 1.6 The stability index of a local effectivity function $E$ is equal to the minimal length of a cycle of $E$ (with the convention that this number is $+\infty$ if $E$ has no cycle)

\section{The meet game form}

A partially ordered set, or poset, is a pair $(A, \geq)$ where $\geq$ is a binary relation on $A$ that is reflexive, transitive and antisymmetric. A poset is a meet semilattice if any pair $\{x, y\} \subset A$ has an infimum, that is a greatest lower bound, denoted $x \wedge y$. The infimum of any family $\left(x_{1}, \ldots, x_{k}\right)$ will be denoted $x_{1} \wedge \cdots \wedge x_{k}$. In this section $(A, \geq)$ is assumed to be a finite meet semilattice and $\Gamma=\left\langle X_{1}, \ldots, X_{n}, A, \mu\right\rangle$ is the meet game form (1) defined on $A$. $\Gamma$ has the following remarkable property:

Proposition 2.1 For any $R_{N} \in Q(A)^{N}$, an outcome is an $\mathcal{M}$-equilibrium outcome of $\Gamma$ if and only if it is in the $\mathcal{M}$ - exact core of $\Gamma$ that is :

$$
E O(\mathcal{M})\left(\Gamma, R_{N}\right)=C_{1, \mathcal{M}}\left(\Gamma, R_{N}\right)
$$


Proof. $E O(\mathcal{M})\left(\Gamma, R_{N}\right) \subset C_{1, \mathcal{M}}\left(\Gamma, R_{N}\right)$ for any game form. In order to prove the opposite inclusion, let $a \notin E O(\mathcal{M})\left(\Gamma, R_{N}\right)$. For any $x=\left(x_{1}, \ldots, x_{n}\right)$ such that $\mu(x)=$ $a$, there exists some $S_{x} \in \mathcal{M}$ and $y_{S_{x}}$ such that $\mu\left(x_{S_{x}^{c}}, y_{S_{x}}\right) R_{i}^{\circ} a$ for all $i \in S_{x}$. The main point is to prove that one can choose some "deviation" $\left(S_{x}, y_{S_{x}^{c}}\right)$ that does not depend on $x$. Let $S$ be the coalition corresponding to $\bar{x}=(a, \ldots, a)$ and let $b=\mu\left(\bar{x}_{S^{c}}, y_{S}\right)$. One has $b R_{i}^{\circ} a$ for all $i \in S$. Let $c=\wedge_{i \in S} y_{i}$. Then $a \wedge c=b$. Clearly $b \neq a$. If $S=N$ then for any $x$ such that $\mu(x)=a, \mu\left(y_{N}\right)=b=c$ thus $a \notin C_{1, \mathcal{M}}\left(\Gamma, R_{N}\right)$. If $S \neq N$ then $b<a$. Let $b_{S} \in A^{S}$ with all components equal to $b$. For any $x$ such that $\mu(x)=a$ one has: $b<a \leq \wedge_{j \in S^{c}} x_{j}$. It follows that $\mu\left(x_{S^{c}}, b_{S}\right)=\left(\wedge_{j \in S^{c}} x_{j}\right) \wedge b=b$. Again $a \notin C_{1, \mathcal{M}}\left(\Gamma, R_{N}\right)$.

Corollary 2.2 The meet game form $\Gamma$ is $\mathcal{M}$-solvable if and only if it is $\mathcal{M}$-exactly stable. The stability index of $\Gamma$ is the same whether we consider the $\mathcal{M}$-exact core or the $\mathcal{M}$-equilibrium.

Thus studying stability of the local effectivity function is sufficient not only for $\mathcal{M}$-exact stability of $\Gamma$, but also for its $\mathcal{M}$-solvability. Here is its precise description for any $\mathcal{M}$ :

Proposition 2.3 For $U \in \mathcal{P}_{0}(A)$, one has :

$$
E_{1, \mathcal{M}}^{\Gamma}[U](S)= \begin{cases}\left\{B \in \mathcal{P}_{0}(A) \mid \forall a \in U, \exists b \in B: a \geq b\right\} & \text { if } S \in \mathcal{M}, S \neq N \\ \mathcal{P}_{0}(A) & \text { if } S \in \mathcal{M}, S=N \\ \emptyset & \text { if } S \notin \mathcal{M}\end{cases}
$$

Proof. Since $E_{1, \mathcal{M}}^{\Gamma}[U](S)=\cap_{a \in U} E_{1, \mathcal{M}}^{\Gamma}[a](S)$, it is enough to prove the formula for $E_{1, \mathcal{M}}^{\Gamma}[a](S)(a \in A)$. That $E_{1, \mathcal{M}}^{\Gamma}[a](N)=\mathcal{P}_{0}(A)$ is straightforward. Let $S \in \mathcal{P}_{0}(N)$, $S \neq N$ and let $B \in \mathcal{P}_{0}(A)$ and $b \in A$ such that $b \in B$ and $b \leq a$. Let $b_{S} \in A^{S}$ with all components equal to $b$. For any $x$ such that $\mu(x)=a, b \leq a \leq\left(\wedge_{j \in S^{c}} x_{j}\right)$. Thus $\mu\left(x_{S^{c}}, b_{S}\right)=\left(\wedge_{j \in S^{c}} x_{j}\right) \wedge b=b$. Therefore $B \in E_{1, \mathcal{M}}^{\Gamma}[a](S)$. Conversely if $B \in$ $E_{1, \mathcal{M}}^{\Gamma}[a](S)$, then in particular taking $x=(a, \ldots, a) \in A^{N}$ there exists $y_{S} \in A^{S}$ such that $\mu\left(x_{S}, y_{S^{c}}\right) \in B$. Since $\mu\left(x_{S}, y_{S^{c}}\right) \leq a$, the proof is complete.

As a hint of the technical tools needed for our purpose, one can consider a special kind of cycle as a sequence $a_{1}, S_{1}, a_{2}, S_{2}, \ldots, a_{p+1}$, where $\left\{a_{1}, \ldots, a_{p}\right\}=A$ and $a_{p+1}=$ $a_{1}$, and with the condition that, starting at $a_{k}$, coalition $S_{k}$ can reach $a_{k+1}$. Therefore the following conditions must be satisfied (1) if $S_{k} \in \mathcal{M}$ and $S_{k} \neq N$ then $a_{k+1}<a_{k}$, and (2) the intersection of $S_{1} \ldots, S_{p}$ must be empty (in order to satisfy property (C") of Definition 1.4). We shall refer informally to such a sequence as a circular domination chain. Figure 2 represents a set of 5 alternatives, with a semilattice structure, the strict part of it being represented by arrows. Consider the sequence $a_{1}, S_{1}, a_{2}, \ldots, a_{5}, S_{5}, a_{1}$. Domination is possible only if $S_{1}=S_{2}=S_{3}=S_{4}=N$. Thus $\cap_{k=1}^{k=5} S_{k}=S \neq \emptyset$. It follows that such a sequence is not a cyclic domination chain. Figure 1 represents a sequence in the lattice $\{1,2,3,4\}$ with its natural order : $e_{1}, S_{1}, e_{2}, \ldots, e_{4}, S_{4}, e_{1}$. The latter is a circular domination chain if and only if $S_{4}=N$ and $\cap \cap_{k=1}^{k=3} S_{k}=\emptyset$.

The examples suggest that the parameters involved in the formation of cycles are two-fold. On the player side, chains of coalitions with non empty intersections will play an important role. This is why we introduce the following (Nakamura [7]): 
Definition 2.4 A nonempty subset $\mathcal{T} \subset \mathcal{M}$ has the empty intersection property if $\cap_{S \in \mathcal{T}} S=\emptyset$. The Nakamura Number of $\mathcal{M}$, denoted $\nu_{\mathcal{M}}$, is the minimum of the cardinality of $\mathcal{T}$ where $\mathcal{T}$ describes all the subsets of $\mathcal{M}$ with the empty intersection property (with the convention that this number is $+\infty$ if no subset of $\mathcal{M}$ has the empty intersection property).

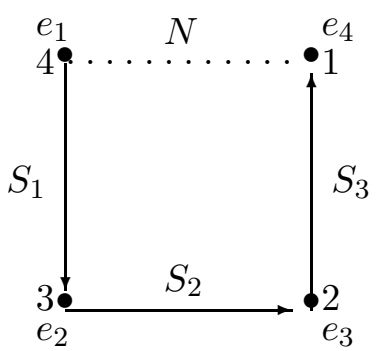

Figure 1: A circular domination chain in the lattice $\mathbb{I}_{4}$ with its natural order: $a \searrow b$ if and only if $a>b$

When it comes to alternatives, one has to take into account the semilattice structure. In a circular chain, any couple $\left(a_{k}, a_{k+1}\right)$ will be called step if $a_{k+1}<a_{k}$ and gap otherwise. In order to characterize stability we shall need to consider chains with the largest number of steps and in order to determine the stability index we shall also need to find, among these chains, one with the smallest number of gaps. In the following subsection we provide the precise definitions that are needed for our characterization.

\subsection{Steps and gaps of a binary relation}

Let $\mathbb{N}^{*}=\{1,2, \ldots\}$ denote the set of strictly positive natural numbers. For $q \in \mathbb{N}^{*}$ let $\mathbb{I}_{q}$ denote the interval $\{1, \ldots, q\}$, and $\mathbb{Z} / q \mathbb{Z}$ the quotient of $\mathbb{Z}$ by the its additive subgroup $q \mathbb{Z}$. Addition in $\mathbb{Z} / q \mathbb{Z}$ is the addition modulo $q$. Let $(A, \searrow)$ be a binary relation on $A$. A couple $(a, b) \in A \times A$ such that $a \searrow b$ will be called a step. Let $q \in \mathbb{N}^{*}$. A $q$-enumeration of $A$ is an injective mapping $e: \mathbb{Z} / q \mathbb{Z} \rightarrow A$. Let $e$ be a $q$-enumeration of $A$. An $e$-edge is any ordered pair of the form $v=\left(e_{k}, e_{k+1}\right)$ where $k \in \mathbb{Z} / q \mathbb{Z}$. Thus a 1-enumeration $e$ has only one edge $\left(e_{1}, e_{1}\right)$. Two $e$-edges $v$ and $w$ are said to be adjacent if $v=\left(e_{k}, e_{k+1}\right)$ and $w=\left(e_{\ell}, e_{\ell+1}\right)$ and $k+1=\ell$. An $e$-chain is any sequence $c=\left(v_{1}, \ldots, v_{r}\right)$ of distinct $e$-edges such that $v_{k}$ and $v_{k+1}$ are adjacent $(k=1, \ldots, r-1)$. The length of $c$ is the number of its $e$-edges. It is denoted $|c|$. Since

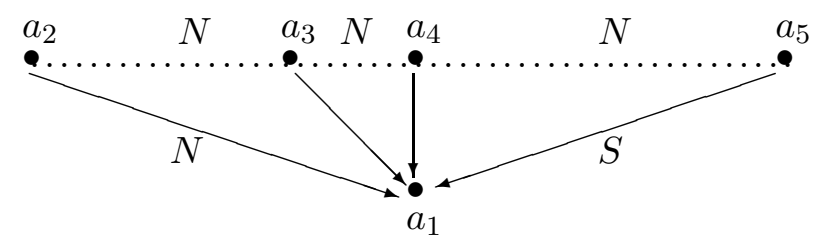

Figure 2: $a \searrow b$ iff there is an arrow from $a$ to $b$. No circular domination chain can be found 
there is no repetition of edges in a chain: $|c| \leq q$. There are exactly $q e$-chains with length $q$, where only the initial vertex differ; we shall identify them all with $e$. An $e$-edge is an $e$-step if it is a step. We usually use the same notation for an $e$-chain (a sequence of e-edges) and the set of its edges. Thus $c \cap c^{\prime}=\emptyset$ means that $c$ and $c^{\prime}$ do not have common edges. Let $c$ and $c^{\prime}$ be two $e$-chains such that $c^{\prime} \subset c$. We say that $c^{\prime}$ is a $c$-gap if $c^{\prime}$ contains no steps and if it is maximal for inclusion in $c$ for this property. If $c$ is an $e$-chain, we denote by $d(c)$ the number of $e$-steps in $c$, and $g(c)$ the number of $c$-gaps. It is easy to see that $d(c)+g(c) \leq|c|$. For $k \geq 1$, let $C_{e}^{k}$ be the set of all $e$-chains such that $d(c)=k$. We introduce the following numbers related to the binary relation $(A, \searrow)$ :

$\delta_{A}=\max _{e} d(e)$ where $e$ describes all the set of $p$-enumerations.

$\gamma_{e}(k)=\min _{c \in C_{e}^{k}} g(c)$ with the convention $\gamma_{e}(k)=+\infty$ if $C_{e}^{k}=\emptyset$.

$\gamma_{A}(k)=\min _{e} \gamma_{e}(k)$ where $e$ describes all the set of $p$-enumerations.

$\delta_{A}$ will be called the depth of $A, \gamma_{e}(\cdot)$ will be called the gap function of $e$ and $\gamma_{A}(\cdot)$ will be call the gap function of $A$. Remark that $\gamma_{e}$ and $\gamma_{A}$ are increasing functions. By convention $\gamma_{e}(+\infty)=\gamma_{A}(+\infty)=+\infty$.

A binary relation $(A, \searrow)$ is said to be acyclic if for any $q \in \mathbb{N}^{*}$, any $q$-enumeration $e$ contains at least one e-gap. If $(A, \searrow)$ is acyclic, then in particular it is irreflexive that is one never has $x \backslash x$ for $x \in A$. To any poset $(A, \geq)$ one can associate the binary relation $(A,>)$ where $x>y$ if and only if $x \geq y$ and $x \neq y .(A,>)$ is then an acyclic binary relation.

Examples 2.5 (a) Let $A=\mathbb{I}_{p}=\{1, \ldots, p\}$ and $a \backslash b$ if and only if $a=b+1$ (addition in $\mathbb{N})$. Then $(A, \searrow)$ is acyclic. Let $e$ be the $p$-enumeration $e(k)=p-k+1(\bmod p)$ then $\delta_{A}=d(e)=p-1 . \gamma_{e}(k)=0$ if $1 \leq k<p, \gamma_{e}(k)=+\infty$ if $k \geq p . \gamma_{A}=\gamma_{e}$.

(b) Let $A=\mathbb{Z} / p \mathbb{Z}(p \geq 2)$ and $a \searrow b$ if and only if $a=b+1$ (addition in $\mathbb{Z} / p \mathbb{Z}$ ). Then $(A, \searrow)$ is not acyclic. Let $e$ be the $p$-enumeration $e(k)=p-k+1$ then $\delta_{A}=d(e)=p$. $\gamma_{e}(k)=0$ if $1 \leq k \leq p, \gamma_{e}(k)=+\infty$ if $k>p . \gamma_{A}=\gamma_{e}$.

(c) Let $A=\mathbb{I}_{p}(p \geq 2)$ and $a \searrow b$ if and only if $a \neq 1$ and $b=1$ or $a=b$. Then $(A$, \) is a meet semilattice, hence acyclic. Let $e$ be the $p$-enumeration $e(k)=p-k+1$ then $\delta_{A}=d(e)=1 . \gamma_{e}(k)=1$ if $k=1, \gamma_{e}(k)=+\infty$ if $k>1 . \gamma_{A}=\gamma_{e}$.

(d) Let $A=\left(\mathbb{I}_{m}\right)^{d}$ and $\left(a_{1}, a_{2}, \ldots, a_{d}\right) \geq\left(b_{1}, b_{2}, \ldots, b_{d}\right)$ if and only if $a_{i} \geq b_{i}(i=$ $1, \ldots, d)$. Then $(A, \geq)$ is a lattice. What are $\delta_{A}$ and $\gamma_{A}$ ?

Proposition 2.6 Let $(A, \searrow)$ be an acyclic binary relation, and let $k \geq 1$. Then:

(i) $\delta_{A}$ is the maximum of $d(e)$ where $e$ describes the union of all $q$-enumerations $(q=$ $1, \ldots, p)$.

(ii) $\gamma_{A}(k)$ is the minimum of $\gamma_{e}(k)$ where $e$ describes the union of all q-enumerations $(q=1, \ldots, p)$.

Proof. See appendix.

We are now ready to state the main results on stability and stability index of the meet game form: 
Theorem 2.7 For any $\mathcal{M}$, the meet game form $\Gamma$ is $\mathcal{M}$ - $\beta$-stable. $\Gamma$ is $\mathcal{M}$-exactly stable (and therefore $\mathcal{M}$-solvable) if and only if either $N \notin \mathcal{M}$ or $\delta_{A}<\nu_{\mathcal{M}}$.

In particular the meet game form is Nash solvable. If $n \geq 2$ and $\mathcal{M}=\mathcal{P}_{0}(N)$, then $\nu_{\mathcal{M}}=2$. Thus the meet game form is strongly solvable if and only if $\delta_{A}=1$.

Theorem 2.8 If $N \in \mathcal{M}$, the stability index of the meet game form relatively to the $\mathcal{M}$-exact core (and therefore $\mathcal{M}$-equilibrium) is equal to: $\nu_{\mathcal{M}}+\gamma_{A}\left(\nu_{\mathcal{M}}\right)+1$.

In particular, if $n \geq 2$ and $\mathcal{M}=\mathcal{P}_{0}(N)$, the strong Nash stability index of the meet game form is equal to $\gamma_{A}\left(\nu_{\mathcal{M}}\right)+3$. The meet game form corresponding to example 2.5 (c) is similar to the unanimity game form. If all players agree on some alternative, then the latter is chosen, if not, then 1 is chosen. Since $\delta_{A}=1<\nu_{\mathcal{M}}$ the game form is $\mathcal{M}$-solvable. The meet game corresponding to example $2.5(\mathrm{a})$ is not $\mathcal{M}$-solvable if $N \in \mathcal{M}$ and $\nu_{\mathcal{M}} \leq p-1$, and in this case the stability index is equal to $\nu_{\mathcal{M}}+1$ since $\gamma_{A}\left(\nu_{\mathcal{M}}\right)=0$. In the next section we give a proof of both theorems in a slightly more general framework.

\section{Stability and Index of the meet game form}

In this section we assume that $(A, \searrow)$ is an acyclic binary relation. We shall write $(a \searrow b)$ if $(a \searrow b)$ or $(a=b)$. For any $\emptyset \neq \mathcal{M} \subset \mathcal{P}_{0}(N)$, we consider the local effectivity function $E_{\mathcal{M}}$ defined as follows: For $U \in \mathcal{P}_{0}(A)$ :

$$
E_{\mathcal{M}}[U](S)= \begin{cases}\left\{B \in \mathcal{P}_{0}(A) \mid \forall a \in U, \exists b \in B: a \searrow \backslash b\right\} & \text { if } S \in \mathcal{M}, S \neq N \\ \mathcal{P}_{0}(A) & \text { if } S \in \mathcal{M}, S=N \\ \emptyset & \text { if } S \notin \mathcal{M}\end{cases}
$$

The corresponding effectivity function is defined by $E_{0, \mathcal{M}}[U](S)=E_{0, \mathcal{M}}(S)=E_{\mathcal{M}}[A](S)$ $(S \in \mathcal{P}(N))$. Let $A_{0}$ be the set of minimal elements of $(A, \Downarrow): x \in A_{0}$ if and only if there is no $y \in A$ such that $x \backslash y$. Since $A$ is finite and $(A, \searrow)$ acyclic, $A_{0} \neq \emptyset$. It is then easy to see that, for any $S \in \mathcal{M}, S \neq N$ any $B \in E_{0, \mathcal{M}}(S)$ contains $A_{0}$. In the case where $\searrow$ is a transitive, the converse is also true: $B \in E_{0, \mathcal{M}}(S)$ if and only if $A_{0} \subset B$.

Lemma 3.1 Let $\left(U_{1}, \ldots, U_{r}\right)$ be a partition of $A$ and let $\left(B_{1}, \ldots, B_{r}\right)$ be a family of nonempty subsets of $A$. Then there exists a subset $I=\left\{k_{1}, \ldots, k_{s}\right\}$ where $1 \leq s \leq r$ such that $B_{k_{j}} \cap U_{k_{j+1}} \neq \emptyset(j=1, \ldots, s)(\bmod s)$.

Proof. Let $\mathcal{I}$ be the set of nonempty subsets $I \subset \mathbb{I}_{r}$ such that for any $k \in I$ there exists $\ell \in I$ such that $B_{k} \cap U_{\ell} \neq \emptyset$. Clearly $\mathbb{I}_{r} \in \mathcal{I}$. Let $I_{0}$ be a minimal set for inclusion in $\mathcal{I}$. For any $k \in I_{0}$ put $\theta(k)$ one of the indices $l \in I_{0}$ such that $B_{k} \cap U_{\ell} \neq \emptyset$. Take $k_{1} \in I_{0}$ arbitrary and put $k_{j+1}=\theta\left(k_{j}\right) j=1,2, \ldots$ By minimality of $I_{0}$, the sequence $\left(k_{1}, \ldots, k_{s}\right)$ is composed of distinct indices and $k_{s+1}=k_{1}$.

Theorem $3.2 E_{0, \mathcal{M}}$ is stable for any $\mathcal{M}$. 
Proof. Assume that $C\left(E_{0, \mathcal{M}}, R_{N}\right)=\emptyset$ for some $R_{N} \in Q(A)^{N}$. Let $x_{0} \in A_{0}$. Then $P\left(x_{0}, S, R_{N}\right) \in E_{0, \mathcal{M}}(S)$ for some $S \in \mathcal{M}$. In view of the remark preceding Lemma 3.1 , we have $S=N$ : indeed $x_{0} \in A_{0}$ and $x_{0} \notin P\left(x_{0}, S, R_{N}\right)$. Therefore $N \in \mathcal{M}$. Moreover, one can construct by induction a sequence $x_{0}, \ldots, x_{t+1}$ such that $x_{k}$ is Pareto dominated by $x_{k+1}$ for $k=0, \ldots, t-1$ and $x_{t+1}$ not Pareto dominated. Two consequences follow: (1) $x_{t+1} \in P\left(x_{0}, N, R_{N}\right)$ and (2): there exists some $S \in \mathcal{M}$, $S \neq N$ such that $P\left(x_{t+1}, S, R_{N}\right) \in E_{0, \mathcal{M}}(S)$. Since $x_{0} \in A_{0} \subset P\left(x_{t+1}, S, R_{N}\right)$ we have $x_{0} \in P\left(x_{t+1}, S, R_{N}\right)$. The latter contradicts (1).

Theorem $3.3 E_{\mathcal{M}}$ is stable if and only if either $N \notin \mathcal{M}$ or $\delta_{A}<\nu_{\mathcal{M}}$.

Proof. Assume that $E_{\mathcal{M}}$ is not stable. Let $R_{N} \in Q(A)^{N}$ be such that $C\left(E_{\mathcal{M}}, R_{N}\right)$ is empty. Put $A=\left\{a_{1}, \ldots, a_{p}\right\}$. For any $k \in \mathbb{I}_{p}$, there exists $b_{k} \in A$ and $S_{k} \in \mathcal{M}$ such that $\left\{b_{k}\right\} \in E_{\mathcal{M}}\left(S_{k}\right)$, and $b_{k} R_{i}^{\circ} a_{k}$ for all $i \in S_{k}$. Let $U_{k}=\left\{a_{k}\right\}$ and $B_{k}=\left\{b_{k}\right\}$. By Lemma 3.1, there exists a subset $I=\left\{k_{1}, \ldots, k_{s}\right\}$ where $1 \leq s \leq p$ such that $b_{k_{j}}=a_{k_{j+1}}(j=1, \ldots, s)(\bmod s)$. Therefore we have $(1) \cap_{k \in I} S_{k}=\emptyset$. Let $e(j)=b_{k_{j}}$ for all $j \in \mathbb{Z} / s \mathbb{Z}$ and let $J=\left\{k \in \mathbb{I}_{p} \mid S_{k} \neq N\right\}$. For all $k \in J, a_{k} \backslash b_{k}$. Then $e$ is an $s$-enumeration, such that $e(j-1) \searrow e(j)$ if $k_{j} \in J$. It follows first that $I$ is not a subset of $J$, for otherwise $e$ would be a cycle for the binary relation $\searrow$ and the latter is acyclic. Therefore there exists $k \in I$ such that $S_{k}=N$, hence $N \in \mathcal{M}$. It follows also that $|J \cap I| \leq d(e) \leq \delta_{A}$. On the other hand since $e(j) R_{i}^{\circ} e(j-1)$ for all $i \in S_{k_{j}}$ $(\bmod s)$ we have $\cap_{k \in J \cap I} S_{k}=\cap_{k \in I} S_{k}=\emptyset$ (by (1)), so that $\nu_{\mathcal{M}} \leq|J \cap I|$. We conclude that $\nu_{\mathcal{M}} \leq \delta_{A}$.

Conversely, assume that $N \in \mathcal{M}$ and $\nu_{\mathcal{M}} \leq \delta_{A}$. Let $e$ be a $p$-enumeration such that $d(e)=\delta_{A}$. Let $J$ be the set of indices $k \in\{1, \ldots, p\}$ such that $e_{k} \backslash e_{k+1}(\bmod p)$. Then $|J|=\delta_{A}$. Let $I \subset J$ such that $|I|=\nu_{\mathcal{M}}$. Let $\left(T_{k}, k \in I\right)$ be a family of elements of $\mathcal{M}$ such that $\cap_{k \in I} T_{k}=\emptyset$. We consider the $p$-tuple $F=\left(\left(U_{1}, B_{1}, S_{1}\right), \ldots,\left(U_{p}, B_{p}, S_{p}\right)\right)$ defined as follows: For $k \in\{1, \ldots, p\}, U_{k}:=\left\{e_{k}\right\}, B_{k}:=\left\{e_{k+1}\right\}(\bmod p)$; if $k \in I$, $S_{k}:=T_{k}$ and if $k \notin I, S_{k}:=N$. Since $B_{k} \in E\left[U_{k}\right]\left(S_{k}\right)$ for all $k \in \mathbb{I}_{p}, F$ is a dominance configuration. We now show that this configuration verifies property $(\mathrm{C})$ of definition 1.4. Let $K$ be any subset of $\{1, \ldots, p\}$ such that $\cap_{k \in K} S_{k} \neq \emptyset$. There exists some $k_{0} \in I$ such that $k_{0} \notin K$. Let $k_{1}$ be the closest index in $K$ that appears after $k_{0}(\bmod p)$. Thus $\left\{k_{0}, \ldots, k_{1}-1\right\}$ is an interval in $\mathbb{Z} / q \mathbb{Z}$, that contains $k_{0}$ and that has empty intersection with $K$. Since $k_{1}-1 \notin K$, we have that $e_{k_{1}} \notin\left\{e_{k+1}: k \in K\right\}$. Since $U_{k_{1}}=\left\{e_{k_{1}}\right\}$ and $\cup_{k \in K} B_{k}=\left\{e_{k+1}: k \in K\right\}$, we have proved $U_{k_{1}} \cap\left(\cup_{k \in K} B_{k}\right)=\emptyset$. Thus $F$ is a cycle.

Theorem 3.4 Assume $N \in \mathcal{M}$. We have the equality: $\sigma\left(E_{\mathcal{M}}\right)=\nu_{\mathcal{M}}+\gamma_{A}\left(\nu_{\mathcal{M}}\right)+1$.

Proof. We first consider the particular case where $\nu_{\mathcal{M}}>\delta_{A}$. In view of Theorem 3.3, $E_{\mathcal{M}}$ is stable, so that $\sigma=+\infty$. If $\nu_{\mathcal{M}}=+\infty$ then the equality is verified. If $\nu_{\mathcal{M}}<+\infty$ then by definition, since $\nu_{\mathcal{M}}>\delta_{A}$, one has $\gamma_{A}\left(\nu_{\mathcal{M}}\right)=+\infty$. Again the equality is verified.

Assume that $\nu_{\mathcal{M}} \leq \delta_{A}$. Let $F=\left(\left(U_{1}, B_{1}, S_{1}\right), \ldots,\left(U_{r}, B_{r}, S_{r}\right)\right)$ be a cycle. We are going to prove $r \geq \nu_{\mathcal{M}}+\gamma_{A}\left(\nu_{\mathcal{M}}\right)+1$. In view of the structure of $E_{\mathcal{M}}$ there exists $\varphi: A \rightarrow A$ with the following properties: (1) $x \in U_{k} \Rightarrow \varphi(x) \in B_{k}$ and (2) $T_{k} \neq N \Rightarrow x \backslash \varphi(x)$. Since the set of minimal elements $A_{0}$ is non empty we choose $x_{1} \in A_{0}$. We construct a sequence $\left(x_{k}\right)$ in $A$ by induction by putting $x_{k+1}=\varphi\left(x_{k}\right) k=1,2, \ldots$, and a sequence 
$\left(t_{k}\right)$ in $\mathbb{I}_{r}$ by defining $t_{k}$ as the unique element in $\mathbb{I}_{r}$ such that $x_{k} \in U_{t_{k}}$. Let $k_{1} \in \mathbb{N}^{*}$ be the smallest integer such that there exists $k_{2} \in \mathbb{N}^{*}, k_{2}>k_{1}$ and $t_{k_{1}}=t_{k_{2}}$. Clearly $\left(t_{1}, \ldots, t_{k_{2}-1}\right)$ are all distinct. Therefore $k_{2}-1 \leq r$. We distinguish 4 cases:

Case 1. $k_{1}>1$ and $x_{k_{2}} \neq x_{k_{1}}$. We put $c=\left(x_{k_{1}}, \ldots, x_{k_{2}}\right)$ and $e=\left(x_{k_{1}}, \ldots, x_{k_{2}}, x_{k_{1}}\right)$. e is a $q$-enumeration, $c$ is an $e$-chain and $q=k_{2}-k_{1}+1$. Thus $q \leq(r+1)-2+1=r$.

Case 2. $k_{1}>1$ and $x_{k_{2}}=x_{k_{1}}$. We put $c=e=\left(x_{k_{1}}, \ldots, x_{k_{2}-1}, x_{k_{2}}\right)$ this is a $q$ enumeration with $q \leq r-1$.

Case 3. $k_{1}=1$ and $x_{k_{2}} \neq x_{k_{1}}$. We put $c=\left(x_{k_{1}}, \ldots, x_{k_{2}}\right)$ and $e=\left(x_{k_{1}}, \ldots, x_{k_{2}}, x_{k_{1}}\right) . e$ is a $q$-enumeration, $c$ is an $e$-chain and $q \leq(r+1)-1+1=r+1$. Since $x_{k_{1}}=x_{1} \in A_{0}$, $\left(x_{k_{1}}, x_{k_{1}+1}\right)$ is not a step.

Case 4. $k_{1}=1$ and $x_{k_{2}}=x_{k_{1}}$. We put $c=e=\left(x_{k_{1}}, \ldots, x_{k_{2}-1}, x_{k_{2}}\right)$ this is a $q$ enumeration with $q \leq r$.

First we establish a lower bound on the depth of $c$. Precisely we prove:

Claim. $d(c) \geq \nu_{\mathcal{M}}$.

Prove of the claim. Put $I=\left\{k_{1}, \ldots, k_{2}-1\right\}, J=\left\{k \in I \mid S_{t_{k}} \neq N\right\}$. We claim that: $\cap_{k \in J} S_{t_{k}}=\emptyset$. The proof is by contradiction: Assume that $\cap_{k \in I} S_{t_{k}}=\cap_{k \in J} S_{t_{k}} \neq \emptyset$, then by property (C) of cycles there exists $\ell \in I$ such that for all $k \in I: U_{t_{\ell}} \cap B_{t_{k}}=\emptyset$. If $\ell \neq k_{1}, x_{\ell} \in U_{t_{\ell}}$ and $x_{\ell}=\varphi\left(x_{\ell-1}\right) \in B_{t_{\ell-1}}$, a contradiction. If $\ell=k_{1}$, then in cases 2 and $4, x_{k_{1}} \in U_{t_{1}}$ and $x_{k_{1}}=x_{k_{2}} \in \varphi\left(x_{k_{2}-1}\right) \in B_{t_{k_{2}-1}}$, a contradiction, and in cases 1 and $3, x_{k_{2}} \in U_{t_{k_{2}}}=U_{t_{k_{1}}}$ and $x_{k_{2}} \in \varphi\left(x_{k_{2}-1}\right) \in B_{t_{k_{2}-1}}$, again a contradiction. Thus we proved $\cap_{k \in J} S_{t_{k}}=\emptyset$. It follows that $|J| \geq \nu_{\mathcal{M}}$. Put $v_{k}=\left(x_{k}, x_{k+1}\right)(k \in I)$. For any $k \in J, v_{k}$ is a step. Therefore $d(c) \geq|J|$. Thus $d(c) \geq \nu_{\mathcal{M}}$, and our claim is proved.

Now, we establish a lower bound on the number of gaps in $e$ and conclude by the desired inequality.

Cases 2 and 4. Here $c=e$. Using lemma A.2 for the first inequality and monotonicity of $\gamma_{A}$ for the third, one has:

$$
\begin{aligned}
& \quad g(e) \geq \gamma_{e}(d(e))+1 \geq \gamma_{A}\left(d(e)+1 \geq \gamma_{A}\left(\nu_{\mathcal{M}}\right)+1\right. \\
& q=|e| \geq d(e)+g(e) \geq \nu_{\mathcal{M}}+\gamma_{A}\left(\nu_{\mathcal{M}}\right)+1 \\
& \text { Moreover } r \geq q \text {, therefore }: r \geq \nu_{\mathcal{M}}+\gamma_{A}\left(\nu_{\mathcal{M}}\right)+1 .
\end{aligned}
$$

Cases 1 and 3. Here $c$ is an $e$-chain, $c=e \backslash\left(x_{k_{2}}, x_{k_{1}}\right)$.

$$
g(c) \geq \gamma_{e}(d(c)) \geq \gamma_{A}\left(d(c) \geq \gamma_{A}\left(\nu_{\mathcal{M}}\right) .\right.
$$

Case 1. $|c| \geq d(c)+g(c) \geq \nu_{\mathcal{M}}+\gamma_{A}\left(\nu_{\mathcal{M}}\right)$

$$
r \geq q=|c|+1 \geq \nu_{\mathcal{M}}+\gamma_{A}\left(\nu_{\mathcal{M}}\right)+1 \text {. }
$$

Case 3. Here $v_{k_{1}}=\left(x_{k_{1}}, x_{k_{1}+1}\right)$ is not a step. Let $c^{\prime}=c \backslash\left\{v_{k_{1}}\right\}$. We have $d\left(c^{\prime}\right)=d(c)$.

$$
\begin{aligned}
& |c|-1=\left|c^{\prime}\right| \geq d\left(c^{\prime}\right)+g\left(c^{\prime}\right)=d(c)+g\left(c^{\prime}\right) \geq d(c)+\gamma_{e}(d(c) \\
& |c| \geq d(c)+\gamma_{e}\left(d(c)+1 \geq \nu_{\mathcal{M}}+\gamma_{A}\left(\nu_{\mathcal{M}}\right)+1 .\right.
\end{aligned}
$$

Since $q=|c|+1$ and $r \geq q-1$, we have: $r \geq \nu_{\mathcal{M}}+\gamma_{A}\left(\nu_{\mathcal{M}}\right)+1$.

In conclusion we have in all cases the inequality $r \geq \nu_{\mathcal{M}}+\gamma_{A}\left(\nu_{\mathcal{M}}\right)+1$. Thus $\sigma\left(E_{\mathcal{M}}\right) \geq$ $\nu_{\mathcal{M}}+\gamma_{A}\left(\nu_{\mathcal{M}}\right)+1$. This ends the first part of the proof. 
Conversely let $r=\nu_{\mathcal{M}}+\gamma_{A}\left(\nu_{\mathcal{M}}\right)+1$. We are going to construct a cycle of order $r$. Let $e$ be a $p$-enumeration and let $c$ be an $e$-chain such that $d(c)=\nu_{\mathcal{M}}$ and $g(c)=\gamma_{A}\left(\nu_{\mathcal{M}}\right)$. It follows that $\nu_{\mathcal{M}}+\gamma_{A}\left(\nu_{\mathcal{M}}\right) \leq|c|$. Let $q=|c|+1$. In view of lemma A.2, $\bar{c}$, the complement of $c$ in $e$ contains at least an edge that is not a step. Thus $q \leq p$. Without loss of generality let $c=\left(e_{1}, \ldots, e_{q}\right)$. Since $r-1=d(c)+g(c)$, we can write $c$ as a sequence of $e$-steps and $e$-gaps $\left(h_{1}, \ldots, h_{r-1}\right)$. Moreover we put $h_{r}=\left(e_{q}, \ldots, e_{p}, e_{1}\right)$. Let $J$ be the set of indices $k \in\{1, \ldots, r-1\}$ such that $h_{k}$ is a step. Let $\left(T_{k}, k \in J\right)$ a family of elements in $\mathcal{M} \backslash\{N\}$ such that $\cap_{k \in J} T_{k}=\emptyset$. Let $f\left(h_{k}\right)$ (resp. [ $\left.h_{k}\right]$ ) be the final node (resp. the set of nodes) of $h_{k}\left(k \in \mathbb{I}_{r}\right)$. In particular: $f\left(h_{r}\right)=e_{1}$. Let $U_{k}=\left[h_{k}\right] \backslash\left\{f\left(h_{k}\right)\right\}, B_{k}=\left\{f\left(h_{k}\right)\right\}\left(k \in \mathbb{I}_{r}\right)$. Let $S_{k}=T_{k}$ for all $k \in J$ and $S_{k}=N$ for all $k \in \mathbb{I}_{r} \backslash J$. We claim that $F=\left(\left(U_{1}, B_{1}, S_{1}\right), \ldots,\left(U_{r}, B_{r}, S_{r}\right)\right)$ is a cycle of $E_{\mathcal{M}}$. The only point that we need to verify is property (C) of definition 1.4. Let $K \subset \mathbb{I}_{r}$ such that $\cap_{k \in K} S_{k} \neq \emptyset$. There exists some $k_{0} \in J \backslash K$. Let $\ell$ be the first index that comes after $k_{0}(\bmod r)$ such that $\ell \in K$. One has $\cup_{k \in K} B_{k}=\left\{f\left(h_{k}\right): k \in K\right\}$. Moreover $U_{\ell} \equiv\left[h_{\ell}\right] \backslash\left\{f\left(h_{\ell}\right)\right\}$ is an $e$-chain. Let $a \in U_{\ell}$. If $a$ is the first element of the $e$-chain $U_{\ell}$, then $a=f\left(h_{\ell-1}\right)$ and since $\ell-1 \notin K$, one has $a \notin \cup_{k \in K} B_{k}$. If $a$ is not the first element, then there is no $k \in\{1 \ldots, r\}$ such that $a=f\left(h_{k}\right)$. It follows that $U_{\ell} \cap\left(\cup_{k \in K} B_{k}\right)=\emptyset$. This shows that $F$ is a cycle of order $r$. We conclude that $\sigma\left(E_{\mathcal{M}}\right) \leq \nu_{\mathcal{M}}+\gamma_{A}\left(\nu_{\mathcal{M}}\right)+1$. The proof is complete.

\section{Appendix}

\section{A Proof of proposition 2.6}

Definition A.1 Let $(A, \searrow)$ be a binary relation, let $e$ be a $q$-enumeration. A $p$ enumeration $\tilde{e}$ is an extension of $e$ if there exists $k \in \mathbb{I}_{q}$ such that $\left(e_{k+1}, e_{k+2}, \ldots, e_{k+q}\right)$ $(\bmod q)$ is an $\tilde{e}$-chain.

Lemma A.2 Let $(A, \searrow)$ be a binary relation. Let e be a q-enumeration containing some gap $h$. For any e-chain $c$ there corresponds some e-chain $c^{\prime}$ such that $d\left(c^{\prime}\right)=d(c)$ and $c^{\prime} \cap h=\emptyset$. For any $k \geq 1$, any e-chain $c$ such that $g(c)=\gamma_{e}(k)$ leaves some gap in its complement. In particular : $g(c)<g(e)$.

Proof. All the $e$-steps are in the complement of $h$, in particular the $e$-steps of $c$. Thus one can find $c^{\prime}$ in the complement of $h$ with the same $e$-steps that belong to $c$ (but with possibly different gaps). It follows in particular that $g\left(c^{\prime}\right)<g(e)$. Any $e$-chain $c$ such that $g(c)=\gamma_{e}(k)$ must leave some gap in his complement, otherwise $g(e)=g(c)$. Now by the first part of the proof there would exist $c^{\prime}$ such $g\left(c^{\prime}\right)<g(e)$, this contradicts the inequality $g(c) \leq g\left(c^{\prime}\right)$ implied by the definition of $\gamma_{e}(k)$.

Lemma A.3 Let $(A, \searrow)$ be a binary relation. Let e be a q-enumeration containing some gap $h$ and some chain $c$ that do not intersect. Then there exists an extension $\tilde{e}$ of e such that $c$ is an $\tilde{e}$-chain.

Proof. Let $B=A \backslash\left\{e_{1}, \ldots, e_{q}\right\}$. Then $|B|=p-q$. Let $f$ be any bijection between $\mathbb{I}_{p-q}$ and $B$. Without loss of generality let $\left(e_{q}, e_{1}\right)$ be some $e$-edge of $h$. One can define 
$\tilde{e}$ as follows: $\tilde{e}(\ell)=e(\ell)$ for $\ell \in \mathbb{I}_{q}$ and $\tilde{e}(q+k)=f(k)$ for any $k \in \mathbb{I}_{p-q}$. It is clear that $c$ is an $\tilde{e}$-chain.

Proof of Proposition 2.6. (i) If $e$ is a $q$-enumeration, such that $d(e)$ achieves the maximum defined in the statement, then, in view of the acyclicity of the binary relation and lemma A.2, there exists some $e$-chain $c$ of $e$ and some $e$-gap $h$ such that $d(c)=d(e)$ and $h$ does not interset $c$. In view of lemma A.3, there is an extension $\tilde{e}$ of $e$ such that $c$ is a chain of $\tilde{e}$. Since the number of steps of $c$ is the same in $e$ and $\tilde{e}$. This proves (i).

Proof of (ii). If $c$ is some $e$-chain where $e$ is a $q$-enumeration, such that $g(c)$ achieves the minimum defined in the statement, then, in view of lemma A.2, there exists some $e$-gap $h$ that does not intersect $c$. In view of lemma A.3, there is an extension $\tilde{e}$ of $e$ such that $c$ is a chain of $\tilde{e}$. Since the number of steps and gaps in $c$ remain the same, (ii) is proved.

\section{References}

[1] J. Abdou, Exact stability and its applications to strong solvability, Math. Soc. Sci. 39 (2000) 263-275.

[2] J. Abdou, A stability index for local effectivity functions, Math. Social Sciences 59 (2010) 306-313.

[3] J. Abdou, The structure of unstable power mechanisms, Economic Theory, online first, 24 september 2010.

[4] J. Abdou, H. Keiding, On necessary and sufficient conditions for solvability of game forms, Math. Social Sciences 46 (2003) 243-260.

[5] H. Keiding, Necessary and sufficient conditions for stability of effectivity functions, Int. J. Game Theory 14 (1985) 93-101.

[6] H. Moulin, B. Peleg, Cores of effectivity functions and implementation theory, J. Math. Econ. 10 (1982) 115-162.

[7] K. Nakamura, The vetoers in a simple game with ordinal preferences, Int. J. Game Theory 8, issue 1 (1979) 55-61.

[8] B. Peleg, Game theoretic analysis of voting in committees, Cambridge University Press, Gambridge, 1984. 